\title{
Large economies with differential information and without free disposal th
}

\author{
V. Filipe Martins-da-Rocha* \\ Graduate School of Economics, Getulio Vargas Foundation \\ Praia de Botafogo 190, 22250-900 Rio de Janeiro - RJ, Brazil \\ Laura Angeloni \\ Dipartimento di Matematica e Informatica, Università degli Studi di Perugia \\ Via Vanvitelli 1, 06123 Perugia, Italy
}

\begin{abstract}
We consider exchange economies with a continuum of agents and differential information about finitely many states of nature. It was proved in Einy, Moreno, and Shitovitz (2001) that if we allow for free disposal in the market clearing (feasibility) constraints then an irreducible economy has a competitive (or Walrasian expectations) equilibrium, and moreover, the set of competitive equilibrium allocations coincides with the private core. However when feasibility is defined with free disposal, competitive equilibrium allocations may not be incentive compatible and contracts may not be enforceable (see e.g. Glycopantis, Muir, and Yannelis (2002)). This is the main motivation for considering equilibrium solutions with exact feasibility. We first prove that the results in Einy, Moreno, and Shitovitz (2001) are still valid without free-disposal. Then, motivated by the issue of contracts' execution, we adapt the incentive compatibility property introduced in Krasa and Yannelis (1994) and we prove that every Pareto optimal exact feasible allocation is incentive compatible, implying that contracts of competitive or core allocations are enforceable.
\end{abstract}

JEL Classification: D51; D82

Key words: Large exchange economies, Differential information, Competitive and Core allocations, Incentive compatibility

\footnotetext{
The financial support of the GIP ANR (project "croyances") and of the Fondation du Risque (chaire Groupama) is gratefully acknowledged. This work was partially done while V.F. Martins-da-Rocha was visiting the Dipartimento di Matematica e Informatica of the Università degli Studi di Perugia. We thank the audience of the First General Equilibrium Workshop at Rio. Section 6 dealing with contract enforcement and coalitional incentive compatibility has benefited from discussions with J. Correia-da-Silva, W. Daher, F. Forges, C. Hervès-Beloso, E. Moreno-García, K. Podczeck, Y. Vailakis and N. C. Yannelis.

${ }^{*}$ Corresponding author

Email addresses: victor.rocha@fgv.br (V. Filipe Martins-da-Rocha), angeloni@dipmat.unipg.it (Laura Angeloni)
} 


\section{Introduction}

Radner (1968) introduced differential information in the general equilibrium model of Arrow and Debreu (1954). He considers an economy which extends over two time periods with uncertainty at the second period represented by a finite set of states of nature. Each agent is characterized by a random initial endowment, a preference relation on contingent consumption plans and a private information. The private information is represented by a partition of the set of states. At the second period, a state of nature is realized but each agent has incomplete information in the sense that he only knows to which atom of his partition the true state belongs but he cannot discriminate states inside this atom. At the first period, a complete set of contingent contracts is available for trade and before they obtain any information about the realized state of nature, agents arrange contracts which are assumed to be consistent with respect to their private information. At the second period, uncertainty is resolved, information is observed, contracts are executed and consumption takes place.

In this framework, Radner (1968) introduced a competitive equilibrium concept (Walrasian expectations equilibrium) which was presented as an analogue concept to the Walrasian equilibrium in Arrow-Debreu model with complete (symmetric) information. He proved that, under standard assumptions (similar to those used in the existence results by Arrow and Debreu (1954)) on agents' characteristics, a Walrasian expectations equilibrium always exists. Recently, this existence result was generalized in several directions: infinitely many commodities (see Podczeck and Yannelis (2007)), infinitely many states (see HervèsBeloso, Martins-da-Rocha, and Monteiro (2007)) and unbounded consumption sets (see Daher, Martins-da-Rocha, and Vailakis (2007)).

Yannelis (1991) introduced a cooperative equilibrium concept, called the private core, which is an analogue concept to the core for an economy with complete (and symmetric) information, and proved that under appropriate assumptions, the private core is always non-empty. In the definition of the private core, when a coalition blocks an allocation, each member in the coalition uses only his own private information. This cooperative concept of equilibrium has some interesting properties: Koutsougeras and Yannelis (1993) proved that allowing individuals to make redistributions of their initial endowments, based only on their own private information, results in equilibrium allocations that are always Bayesian incentive compatible and also takes into account the informational advantage of an individual. The private core is the appropriate notion of core when the traders do not want to exchange information or when they do not have access to any communication system. When traders are allowed to fully share their information, the appropriate core concept is the weak fine core introduced by Yannelis (1991) (see also Koutsougeras and Yannelis (1993) and Glycopantis and Yannelis (2005)).

For economies with complete information, Aumann (1964) proved that competitive and core allocations coincide, provided that there is a continuum of traders. The existence of such allocations was studied by Aumann (1966) and Hildenbrand (1970). An extension of these results to economies with differential information was proposed by Einy, Moreno, and Shitovitz (2001). They show that, if an economy is irreducible, then a competitive (or Walrasian expectations) equilibrium exists and, moreover, the set of competitive equilibrium allocations coincides with the private core. However, to obtain these results they allow for free disposal on the feasibility (market clearing) constraints. This was motivated by an example provided by Einy and Shitovitz (2001) of an economy with differential information which has a competitive equilibrium with free disposal, but if the feasibility constraints are 
imposed with an equality, then the economy does not have a competitive equilibrium where prices of all contingent contracts for future delivery are non-negative. We claim that this is not economically inconsistent. If there is a state $s$ that no agent can identify, then the contract delivering one unit of a good $\ell$ contingent to the realization of the state $s$ cannot be purchased by any trader. The fact the price $p(s, \ell)$ may be negative is irrelevant, what matter are prices of tradeable contracts. Another reason for considering feasibility constraints with free disposal is the version of Fatou's Lemma used in Hildenbrand (1970) to prove existence of competitive equilibrium. There, arguments are based on a version of Fatou's Lemma proved by Schmeidler (1970) where free disposal plays a crucial role.

The aim of this paper is to investigate whether the results in Einy, Moreno, and Shitovitz (2001) are still valid if the feasibility constraints are imposed with equality. Using a more general version of Fatou's Lemma (proved by Balder and Hess (1995)) and a generalization of Hildenbrand's result by Cornet, Topuzu, and Yildiz (2003), we prove that if an economy is irreducible, then a competitive (or Walrasian expectations) equilibrium exists and moreover, the set of competitive equilibrium allocations coincides with the private core. We also deal with another issue: contracts enforcement at the second period. There is a detailed discussion in Daher, Martins-da-Rocha, and Vailakis (2007) (see also (Podczeck and Yannelis, 2007, Section 4)) about the relationship between the execution of contracts and incentive compatibility properties. When free disposal is allowed, Radner (1968) himself realized that this assumption may be problematic in the context of asymmetric information. Indeed, the total amount to be disposed of might not be measurable with respect to the information partition of a single agent. ${ }^{1}$ This is the main reason why competitive allocations with free disposal may not be incentive compatible (see Glycopantis, Muir, and Yannelis (2002) for an example). We adapt the notion of coalitional incentive compatibility introduced by Krasa and Yannelis (1994) and we prove that every Pareto optimal allocation, satisfying feasibility constraints with equality is coalitional incentive compatible, implying that contracts of a competitive or core allocations are enforceable.

The structure of the paper is as follows. Section 2 presents the theoretical framework and outlines the basic model. In Section 3, we introduce assumptions under which existence of competitive allocations and core equivalence will be proved. The proofs follow in Section 4 and Section 5 respectively. Section 6 is devoted to the issue of contract enforceability and its relationship with coalitional incentive compatibility.

\section{The model}

We consider a pure exchange economy with a continuum of agents represented by a finite positive measure space $(T, \Sigma, \mu)$, where $T$ is a set which represents agents, $\Sigma$ is a $\sigma$-algebra on $T$ which represents coalitions, and $\mu$ is a non-atomic positive and finite measure on $\Sigma$ satisfying $\mu(T)=1$. If $E \in \Sigma$ is a coalition then $\mu(E)$ represents the fraction of agents which belong to $E$.

The economy extends over two time periods $\tau \in\{0,1\}$. There is uncertainty over the possible state of nature that may realize at $\tau=1$ represented by a finite set $\Omega$. Consumption of a finite set $L$ of goods takes place at $\tau=1$ but agents arrange contingent contracts at $\tau=0$ where there is a complete set of contingent contracts for future delivery of each good.

\footnotetext{
${ }^{1}$ More precisely, the total amount to be disposed of may not coincide with the sum of private measurable contingent contracts.
} 
At $\tau=1$ an agent $t$ has an incomplete information about which state of nature actually occurred. This information is described by a partition $\Pi_{t}$ of $\Omega$ : if $\omega$ is the true state of nature, agent $t$ cannot discriminate the states in the (unique) element of $\Pi_{t}$ containing $\omega$. The $\sigma$ algebra generated by $\Pi_{t}$ is denoted by $\mathscr{F}_{t}$ and we denote by $X_{t}$ the set of $\mathscr{F}_{t}$-measurable functions $x: \Omega \rightarrow \mathbb{R}_{+}^{L}$. For every $\omega \in \Omega$ we let $E_{t}(\omega)$ be the unique atom of $\mathscr{F}_{t}$ (or unique element of $\Pi_{t}$ ) containing $\omega$.

Following the model introduced by Radner $(1968)^{2}$ the information of an agent places a restriction on his feasible trade in the sense that each agent $t$ is constrained to choose a contingent contract $x: \Omega \rightarrow \mathbb{R}_{+}^{L}$ measurable with his private information $\mathscr{F}_{t}$. In other words he chooses plans in the consumption set $X_{t}$. Agent $t$ knows at $\tau=0$ that at $\tau=1$ and state $\omega$ he will have an initial endowment $e_{t}(\omega) \in \mathbb{R}_{+}^{L}$. We assume that he can observe his initial endowment, i.e., the function $e_{t}$ is $\mathscr{F}_{t}$-measurable. The ex-ante preference relation about contingent plans at $\tau=0$ is represented by a correspondence $P_{t}: X_{t} \rightarrow 2^{X_{t}}$. If $x \in X_{t}$ is a contingent plan then $P_{t}(x)$ represents the set of plans $y \in X_{t}$ that are strictly preferred to $x$. An economy $\mathscr{E}$ is then defined by a family

$$
\mathscr{E}=\left(\mathscr{F}_{t}, P_{t}, e_{t}\right)_{t \in T} .
$$

Remark 2.1. An economy is said to have preference relations represented by expected utilities if for each agent $t \in T$, there exist

1. a strictly positive $e^{3}$ probability measure $q_{t}$ on $\Omega$ which represents his prior beliefs, and

2. a state dependent utility function $u_{t}: \Omega \times \mathbb{R}_{+}^{L} \rightarrow \mathbb{R}$ satisfying the following properties

(a) the mapping $t \mapsto q_{t}(\omega)$ is $\Sigma$-measurable for each state $\omega \in \Omega ;{ }^{4}$

(b) the mapping $(t, x) \mapsto u_{t}(\omega, x)$ is $\Sigma \times \mathscr{B}\left(\mathbb{R}_{+}^{L}\right)$-measurable;

(c) the mapping $\omega \mapsto u_{t}(\omega, x)$ is $\mathscr{F}_{t}$-measurable for every $x \in \mathbb{R}_{+}^{L}$;

(d) the mapping $x \mapsto u_{t}(\omega, x)$ is continuous and strictly increasing ${ }^{5}$ on $\mathbb{R}_{+}^{L}$;

such that

$$
\forall x \in X_{t}, \quad P_{t}(x)=\left\{y \in X_{t}: h_{t}(y)>h_{t}(x)\right\}
$$

where

$$
h_{t}(x):=\sum_{\omega \in \Omega} q_{t}(\omega) u_{t}(\omega, x(\omega)) .
$$

Since the space $\Omega$ is finite, there exists a finite collection $\left(\mathscr{F}^{i}\right)_{i \in I}$ of $\sigma$-algebras on $\Omega$ such that

$$
\left\{\mathscr{F}_{t}: t \in T\right\}=\left\{\mathscr{F}^{i}: i \in I\right\} .
$$

We assume that the set $T^{i} \subset T$ defined by

$$
T^{i}:=\left\{t \in T: \mathscr{F}_{t}=\mathscr{F}^{i}\right\}
$$

belongs to $\mathscr{T}$ and that the family $\left(T^{i}\right)_{i \in I}$ forms a partition of $T$ satisfying $\mu\left(T^{i}\right)>0$ for each $i$. Therefore there is a finite set $I$ of information types and every agent $t \in T^{i}$ is of information type $i$ in the sense that $\mathscr{F}_{t}=\mathscr{F}^{i}$.

\footnotetext{
${ }^{2}$ We also refer to Radner (1982).

${ }^{3}$ In the sense that for each $\omega \in \Omega$, we have $q_{t}(\omega)>0$.

${ }^{4}$ We abuse notation writing $q_{t}(\omega)$ instead of $q_{t}\{\omega\}$.

${ }^{5} \mathrm{~A}$ mapping $f: \mathbb{R}_{+}^{L} \rightarrow \mathbb{R}$ is strictly increasing if $f(x+y)>f(x)$ for every $x, y \in \mathbb{R}_{+}^{L}$ with $y \neq 0$.
} 
Throughout the paper we use the following notations. For each $i \in I$, the space of $\mathscr{F}^{i}$ measurable functions $x: \Omega \rightarrow \mathbb{R}_{+}^{L}$ is denoted by $E_{+}^{i}$ and the linear space $E_{+}^{i}-E_{+}^{i}$ is denoted by $E^{i}$. Denote by $E_{++}^{i}$ the interior of $E_{+}^{i}$ relative to $E^{i}$, i.e., an $\mathscr{F}^{i}$-measurable function $x$ belongs to $E_{++}^{i}$ if and only if $x(\omega) \in \mathbb{R}_{++}^{L}$ for each state $\omega \in \Omega$. Observe that for each $t \in T^{i}$, the consumption set $X_{t}$ coincides with the set $E_{+}^{i}$. The space $\sum_{i \in I} E^{i}$ is denoted by $E$ and is called the commodity space. The (positive) cone $\sum_{i \in I} E_{+}^{i}$ is denoted by $E_{+}$. Since $E$ may be identified with a vector subspace of $\mathbb{R}^{\Omega \times L}$, it may be endowed with the cone $E \cap \mathbb{R}_{+}^{\Omega \times L}$. Observe that $E_{+}$is a subset of $E \cap \mathbb{R}_{+}^{\Omega \times L}$ but in general, ${ }^{6}$ it is a strict subset. A vector $x \in E_{+}$ is said strictly positive, denoted by $x \gg 0$, if for every $\omega \in \Omega$, the vector $x(\omega)$ belongs to $\mathbb{R}_{++}^{L}$.

Definition 2.1. An integrable function from $T$ to $\mathbb{R}_{+}^{\Omega \times L}$ is called an assignment and the space of assignments is denoted by $\mathscr{S}$. The space of integrable selections of the correspondence $X$, i.e., the space of integrable functions $x: t \mapsto x_{t}$ from $T$ to $E$ such that $x_{t} \in X_{t}$ for $\mu$-a.e. $t \in T$, is denoted by $\mathscr{S}_{X}$. A vector $x$ in $\mathscr{S}_{X}$ is called a private assignment and it is said

1. feasible if

$$
\int_{T} x d \mu=\int_{T} e d \mu
$$

2. free-disposal feasible if

$$
\exists z \in E \cap \mathbb{R}_{+}^{\Omega \times L}, \quad \int_{T} x d \mu+z=\int_{T} e d \mu .
$$

\section{Assumptions}

Throughout the rest of the paper we only consider standard economies in the sense given by the following definition.

Definition 3.1. An economy $\mathscr{E}$ is said standard if

(S.1) the initial endowment assignment $e: t \mapsto e_{t}$ belongs to $\mathscr{S}_{X}$;

(S.2) the aggregate initial endowment $e(T)=\int_{T} e d \mu$ belongs to $\sum_{i \in I} E_{++}^{i}$;

(S.3) preference relations are measurable,${ }^{7}$ irreflexive, ${ }^{8}$ transitive,${ }^{9}$ continuous ${ }^{10}$ and strictly monotone. ${ }^{11}$

\footnotetext{
${ }^{6}$ If for each $\omega \in \Omega$, there exists an information type $i \in I$ such that $\{\omega\}$ belongs to $\mathscr{F}^{i}$, then both cones $E_{+}$and $E \cap \mathbb{R}_{+}^{\Omega \times L}$ coincide.

${ }^{7}$ In the sense that $\left\{(t, x, y) \in T \times E \times E: x, y \in X_{t} \quad\right.$ and $\left.\quad y \in P_{t}(x)\right\} \in \Sigma \times \mathscr{B}(E) \times \mathscr{B}(E)$.

${ }^{8}$ In the sense that for every $x \in X_{t}, x \notin P_{t}(x)$.

${ }^{9}$ In the sense that for every $x, y \in X_{t}$, if $y \in P_{t}(x)$ then $P_{t}(y) \subset P_{t}(x)$.

${ }^{10}$ In the sense that for every $x \in X_{t}$, the set $P_{t}(x)$ and the set $P_{t}^{-1}(x):=\left\{y \in X_{t}: x \in P_{t}(y)\right\}$ are open in $X_{t}$.

${ }^{11}$ In the sense that for every $x, y \in X_{t}$, if $y \neq 0$ then $x+y \in P_{t}(x)$.
} 
Remark 3.1. Conditions (S.1) and (S.3) are standard. Condition (S.2) is satisfied if for each information type $i \in I$, the aggregate initial endowment $e\left(T^{i}\right)$ of the coalition $T^{i}$ is strictly positive, i.e., $e\left(T^{i}\right)(\omega) \in \mathbb{R}_{++}^{L}$ for each $\omega \in \Omega$. When information is symmetric among agents, Condition (S.2) is automatically satisfied if the aggregate initial endowment $e(T)$ is strictly positive, i.e., $e(T)(\omega) \in \mathbb{R}_{++}^{L}$ for each $\omega \in \Omega$.

Remark 3.2. If preference relations are represented by expected utilities then they are automatically measurable, irreflexive, transitive, strictly monotone and continuous.

The following irreducibility condition was introduced in McKenzie (1959) for economies with finitely many agents. It was extended to large economies by Hildenbrand (1974). ${ }^{12}$

Definition 3.2. An economy is said irreducible if for every feasible private assignment $x \in \mathscr{S}_{X}$ and for every two disjoints coalitions $A, B \in \Sigma$ such that $\mu(A)>0, \mu(B)>0$, and $A \cup B=T$, there exist two private assignments $y, z \in \mathscr{S}_{X}$ such that

$$
\int_{A} x d \mu+\int_{B} e d \mu=\int_{A} y d \mu+\int_{B} z d \mu
$$

and

$$
y_{t} \in P_{t}\left(x_{t}\right) \text { for } \mu \text {-a.e. } t \in A \text {. }
$$

We let $\mathscr{F}^{c}:=\bigwedge_{i \in I} \mathscr{F}^{i}$ be the meet ${ }^{13} \sigma$-algebra representing the common knowledge information of the grand coalition $I$, and we denote by $E^{c}\left(E_{+}^{c}\right)$ the space of $\mathscr{F}^{c}$-measurable functions from $\Omega$ to $\mathbb{R}^{L}$ (resp. $\mathbb{R}_{+}^{L}$ ). Observe that $E^{c}$ is a subspace of each $E^{i}$. We propose hereafter a condition on the initial endowment assignment which implies the irreducibility condition.

Proposition 3.1. Let $\mathscr{E}$ be a standard economy such that there exists a private assignment $a \in \mathscr{S}_{X}$ satisfying for $\mu$-a.e. $t \in T$,

$$
0 \neq a_{t} \in E_{+}^{c} \quad \text { and } \quad e_{t}(\omega) \geq a_{t}(\omega), \quad \forall \omega \in \Omega .
$$

Then the economy is irreducible.

Proof. Let $x \in \mathscr{S}_{X}$ be a feasible private assignment and let two disjoints coalitions $A, B \in \Sigma$ such that $\mu(A)>0, \mu(B)>0$, and $A \cup B=T$. We let $a(B):=\int_{B} a d \mu$, observe that $a(B) \in E_{+}^{c}$. We define the function $y: T \rightarrow E$ by

$$
\forall t \in T, \quad y_{t}=\left\{\begin{array}{lll}
x_{t}+\frac{1}{\mu(A)} a(B) & \text { if } & t \in A \\
e_{t} & \text { if } & t \in B .
\end{array}\right.
$$

We define the function $z: T \rightarrow E$ by

$$
\forall t \in T, \quad z_{t}=\left\{\begin{array}{lll}
e_{t} & \text { if } \quad t \in A \\
e_{t}-a_{t} & \text { if } \quad t \in B
\end{array}\right.
$$

\footnotetext{
${ }^{12}$ Einy, Moreno, and Shitovitz (2001) proposed an extension of Hildenbrand's irreducibility condition to economies with differential information which is slightly different from the one we introduced below.

${ }^{13}$ If $J$ is a subset of $I$ then $\bigwedge_{j \in J} \mathscr{F}^{j}$ is the finest $\sigma$-algebra contained in each $\mathscr{F}^{j}, j \in J$.
} 
Observe that the functions $y$ and $z$ belong to $\mathscr{S}_{X}$ and satisfy

$$
\int_{A} x d \mu+\int_{B} e d \mu=\int_{A} y d \mu+\int_{B} z d \mu
$$

and

$$
y_{t} \in P_{t}\left(x_{t}\right) \text { for } \mu \text {-a.e. } t \in A \text {. }
$$

Remark 3.3. Assume that for $\mu$-a.e. $t \in T$, we have $e_{t}(\omega) \in \mathbb{R}_{++}^{L}$ for every $\omega \in \Omega$. Then ${ }^{14}$ there exists a measurable function $\varepsilon(\omega): T \rightarrow(0,1]$ such that $e_{t}(\omega) \geq \varepsilon_{t}(\omega) \mathbf{1}_{L} \cdot{ }^{15}$ Let $a_{t}$ be the vector in $E$ defined by

$$
\forall \omega \in \Omega, \quad a_{t}(\omega)=\left\{\min _{\omega \in \Omega} \varepsilon_{t}(\omega)\right\} \mathbf{1}_{L},
$$

then we have $a_{t} \in E_{+}^{c}, a_{t} \neq 0$ and $e_{t} \geq a_{t}$, implying that the economy is irreducible.

\section{Competitive allocations}

At the first period $\tau=0$ there is a complete set of contingent contracts for future delivery of each good. Therefore a price system is a function $p: \Omega \rightarrow \mathbb{R}^{L}$ where $p(\omega, \ell)$ represents the price at $\tau=0$ of the contract delivering one unit of good $\ell$ if the state of nature at $\tau=1$ is $\omega$. The budget set $B_{t}(p)$ for agent $t$ is then defined by

$$
B_{t}(p):=\left\{x_{t} \in X_{t}: \mathbb{E}\left[p \cdot x_{t}\right] \leqslant \mathbb{E}\left[p \cdot e_{t}\right]\right\}
$$

where

$$
\forall x \in E, \quad \mathbb{E}[p \cdot x]:=\sum_{\omega \in \Omega} p(\omega) \cdot x(\omega) .
$$

We denote by $\mathbb{E}\left[p \mid \mathscr{F}_{t}\right]$ the function from $\Omega$ to $\mathbb{R}_{+}^{L}$ defined by

$$
\forall \omega \in \Omega, \quad \mathbb{E}\left[p \mid \mathscr{F}_{t}\right](\omega)=\frac{1}{\# E_{t}(\omega)} \sum_{\sigma \in E_{t}(\omega)} p(\sigma) .
$$

This function is called the conditional price with respect to $\mathscr{F}_{t}$ or agent $t$ 's conditional price. Since agent $t$ 's choices are constrained by his information, replacing the vector $p$ by the function $\mathbb{E}\left[p \mid \mathscr{F}_{t}\right]$ leads to the same opportunities in the sense that

$$
B_{t}(p)=B_{t}\left(\mathbb{E}\left[p \mid \mathscr{F}_{t}\right]\right) .
$$

Remark 4.1. This does not mean that agent $t$ only observes the price vector $\mathbb{E}\left[p \mid \mathscr{F}_{t}\right]$. Every agent observes the same price vector $p$. However, for agent $t$, the conditional price $\mathbb{E}\left[p \mid \mathscr{F}_{t}\right]$ is as relevant as the price $p$ to make his optimal choice.

\footnotetext{
${ }^{14}$ For each $\omega \in \Omega$, define the function $\varepsilon(\omega)$ by $\varepsilon_{t}(\omega)=\min \left\{1, \min \left\{e_{t}(\omega, \ell): \ell \in L\right\}\right.$, where $e_{t}(\omega, \ell)$ is the $\ell$-th coordinate of $e_{t}(\omega) \in \mathbb{R}^{L}$.

${ }^{15}$ If $A$ is a subset of $L$ then $\mathbf{1}_{A}$ denotes the vector in $\mathbb{R}^{L}$ defined by $\mathbf{1}_{A}(\ell)=1$ if $\ell \in A$ and 0 elsewhere.
} 
In this section we extend to our model the definition of competitive equilibrium (or Walrasian expectations equilibrium) introduced by Radner (1968), and discuss conditions under which its existence can be guaranteed.

Definition 4.1. A pair $(x, p)$ of a private assignment $x \in \mathscr{S}_{X}$ and price $p: \Omega \rightarrow \mathbb{R}^{L}$ is a competitive equilibrium if

(a) for $\mu$-a.e. $t \in T$, the plan $x_{t}$ belongs to the budget set $B_{t}(p)$ and is optimal in the sense that $B_{t}(p) \cap P_{t}\left(x_{t}\right)=\emptyset$; and

(b) $x$ is feasible, i.e., for each possible realization $\omega \in \Omega$, markets clear in the sense that

$$
\forall \omega \in \Omega, \quad \int_{T} x_{t}(\omega) \mu(d t)=\int_{T} e_{t}(\omega) \mu(d t) .
$$

A competitive allocation is a feasible private assignment $x \in \mathscr{S}_{X}$ for which there exists a price $p$ such that $(x, p)$ is a competitive equilibrium.

Remark 4.2. If $(x, p)$ is competitive equilibrium of a standard economy then the following properties are satisfied:

1. for $\mu$-a.e. $t \in T$ the budget set restriction is binding, i.e.,

$$
\mathbb{E}\left[p \cdot x_{t}\right]=\mathbb{E}\left[p \cdot e_{t}\right] ;
$$

2. conditional prices are strictly positive, i.e.,

$$
\forall i \in I, \quad \forall \omega \in \Omega, \quad \mathbb{E}\left[p \mid \mathscr{F}^{i}\right](\omega) \in \mathbb{R}_{++}^{L} .
$$

If for any state $\omega$, the event $\{\omega\}$ belongs to $\mathscr{F}^{i}$ for some agent $i$, then (1) implies that for every $\omega \in \Omega$, the spot price $p(\omega) \in \mathbb{R}_{++}^{L}$. This property is not true in general: (Einy and Shitovitz, 2001, Example 2.1) provide an example of a standard economy with differential information for which there is no competitive equilibrium $(x, p)$ satisfying $p(\omega) \in \mathbb{R}_{+}^{L}$ for every $\omega \in \Omega$.

In Einy, Moreno, and Shitovitz (2001) it is the definition of competitive equilibrium with free-disposal (used by Radner (1982)) that was extended to large economies.

Definition 4.2. A pair $(x, p)$ of a private assignment $x \in \mathscr{S}_{X}$ and price $p: \Omega \rightarrow \mathbb{R}_{+}^{L}$ is a competitive equilibrium with free-disposal if it satisfies the previous property (a) together with the following

(b') $x$ is free disposal feasible, i.e., for each possible realization $\omega \in \Omega$, markets clear with free-disposal in the sense that

$$
\forall \omega \in \Omega, \quad \int_{T} e_{t}(\omega) \mu(d t)-\int_{T} x_{t}(\omega) \mu(d t) \in \mathbb{R}_{+}^{L} .
$$

Remark 4.3. Observe that property (b') can be rewritten as

$$
\exists z \in E \cap \mathbb{R}_{+}^{\Omega \times L}, \quad \int_{T} x_{t} \mu(d t)+z=\int_{T} e_{t} \mu(d t) .
$$

In particular the plan $z$ is not required to be compatible with the information available in the market, i.e., it is not imposed that $z$ belongs to $E_{+}$. Observe moreover that in the definition of a competitive equilibrium, every spot price $p(\omega)$ is required to be nonnegative. 
It was proved in (Einy, Moreno, and Shitovitz, 2001, Theorem A) that every irreducible economy has a competitive equilibrium with free-disposal. We may think that in the context of a pure exchange economy with differential information it is more reasonable to assume inequality in the feasibility constraints since it allows to prove the existence of an equilibrium price $p$ satisfying $p(\omega) \in \mathbb{R}_{+}^{L}$ for each $\omega$. However since each agent $t \in T$ can only make $\mathscr{F}_{t}$-measurable plans, it seems natural to only require that the conditional price $\mathbb{E}\left[p \mid \mathscr{F}_{t}\right]$ with respect to the available information $\mathscr{F}_{t}$ is nonnegative, i.e., for each $t \in T$, the vector $\mathbb{E}\left[p \mid \mathscr{F}_{t}\right](\omega)$ belongs to $\mathbb{R}_{+}^{L}$. To illustrate this point, we propose to consider Example 2.1 in Einy and Shitovitz (2001).

Example 4.1. Consider an economy $\mathscr{E}$ in which the space of traders is $T=[0,3]$ with its Borel subsets and the Lebesgue measure $\lambda$. The set of states of nature is $\Omega=\left\{\omega_{1}, \ldots, \omega_{4}\right\}$. There is only one good, i.e., $L=\{\ell\}$ and the space $\mathbb{R}_{+}^{L}$ is denoted by $\mathbb{R}_{+}$. There are three information types, i.e., $I=\left\{i_{1}, i_{2}, i_{3}\right\}$ where

$$
T^{i_{1}}=[0,1], \quad T^{i_{2}}=(1,2] \quad \text { and } \quad T^{i_{3}}=(2,3] .
$$

Information is defined by

$$
\mathscr{F}^{i_{1}}=\sigma\left(\left\{\omega_{1}, \omega_{2}\right\},\left\{\omega_{3}, \omega_{4}\right\}\right), \quad \mathscr{F}^{i_{2}}=\sigma\left(\left\{\omega_{1}, \omega_{3}\right\},\left\{\omega_{2}, \omega_{4}\right\}\right)
$$

and

$$
\mathscr{F}^{i_{3}}=\sigma\left(\left\{\omega_{1}, \omega_{4}\right\},\left\{\omega_{2}, \omega_{3}\right\}\right) .
$$

All the agents in the economy have the same prior given by

$$
\forall t \in T, \quad q_{t}\left(\omega_{1}\right)=\frac{1}{10} \quad \text { and } \quad q_{t}\left(\omega_{2}\right)=q_{t}\left(\omega_{3}\right)=q_{t}\left(\omega_{4}\right)=\frac{3}{10} .
$$

Random initial endowments are defined by

$$
\begin{aligned}
& \forall t \in T^{i_{1}}, \quad e_{t}(\omega)=\left\{\begin{array}{lll}
101 & \text { if } & \omega \in\left\{\omega_{1}, \omega_{2}\right\} \\
1 / 2 & \text { if } & \omega \in\left\{\omega_{3}, \omega_{4}\right\},
\end{array}\right. \\
& \forall t \in T^{i_{2}}, \quad e_{t}(\omega)=\left\{\begin{array}{lll}
101 & \text { if } & \omega \in\left\{\omega_{1}, \omega_{3}\right\} \\
1 / 2 & \text { if } & \omega \in\left\{\omega_{2}, \omega_{4}\right\},
\end{array}\right.
\end{aligned}
$$

and

$$
\forall t \in T^{i_{3}}, \quad e_{t}(\omega)=\left\{\begin{array}{lll}
101 & \text { if } & \omega \in\left\{\omega_{1}, \omega_{4}\right\} \\
1 / 2 & \text { if } & \omega \in\left\{\omega_{2}, \omega_{3}\right\}
\end{array}\right.
$$

The utility function of each agent is given by

$$
\forall t \in T, \quad \forall \omega \in \Omega, \quad \forall c \geq 0, \quad u_{t}(\omega, c)=\sqrt{c} .
$$

Einy and Shitovitz (2001) proved that there does not exist a competitive equilibrium $(x, p)$ where the price $p$ is such that $p(\omega) \geq 0$ for each $\omega$. However, $(e, \pi)$ is a competitive equilibrium where the price $\pi$ is defined by

$$
\pi\left(\omega_{1}\right)=2 \gamma-1 \quad \text { and } \quad \pi\left(\omega_{2}\right)=\pi\left(\omega_{3}\right)=\pi\left(\omega_{4}\right)=1
$$


where

$$
\gamma=\frac{2}{3} \sqrt{\frac{1 / 2}{101}}
$$

Observe that $\pi\left(\omega_{1}\right)<0$. However, the contract $\mathbf{1}_{\left\{\omega_{1}\right\}}$ delivering one unit of the good contingent to state $\omega_{1}$ cannot be traded by any agent. In particular, no agent can take advantage of this arbitrage opportunity. Indeed, consider for instance an agent $t$ of information type $i_{1}$. He solves the following maximization problem:

$$
\operatorname{argmax}\left\{h_{t}(\alpha, \alpha, \beta, \beta):(\alpha, \beta) \in \mathbb{R}_{+}^{2} \quad \text { and } 2 \gamma \alpha+2 \beta \leqslant 2 \gamma 101+2(1 / 2)\right\} .
$$

In particular, what matters for him is the conditional price $\mathbb{E}\left[\pi \mid \mathscr{F}_{t}\right]$ given by

$$
\mathbb{E}\left[\pi \mid \mathscr{F}_{t}\right](\omega)=\left\{\begin{array}{lll}
\gamma=\frac{\pi\left(\omega_{1}\right)+\pi\left(\omega_{2}\right)}{2} & \text { if } & \omega \in\left\{\omega_{1}, \omega_{2}\right\} \\
1=\frac{\pi\left(\omega_{3}\right)+\pi\left(\omega_{4}\right)}{2} & \text { if } \quad \omega \in\left\{\omega_{3}, \omega_{4}\right\} .
\end{array}\right.
$$

Observe that $\mathbb{E}\left[\pi \mid \mathscr{F}_{t}\right](\omega)>0$ for each $\omega \in \Omega$.

We assert that if we replace the requirement that every equilibrium prices $p(\omega)$ are nonnegative by the requirement that all conditional prices are nonnegative, then it is possible to prove that every irreducible economy has a competitive equilibrium (with an equality in the feasibility constraints).

Theorem 4.1. Every irreducible economy has a competitive equilibrium.

The existence result in Einy, Moreno, and Shitovitz (2001) follows as a corollary of the existence result in Hildenbrand (1974) which is based on a multidimensional Fatou's Lemma provided by Schmeidler (1970). In Schmeidler's version of Fatou's Lemma the positive cone is the lattice cone $\mathbb{R}_{+}^{n}$ of a finite dimensional Euclidean vector space $\mathbb{R}^{n}$. In order to deal with our positive cone $E_{+}$we propose a proof which relies on a generalization of Hildenbrand's existence result provided by Cornet, Topuzu, and Yildiz (2003). The later existence result is based on a generalization of Schmeidler's version of Fatou's Lemma due to Balder and Hess (1995).

Proof of Theorem 4.1. Let $\mathscr{E}=\left(\mathscr{F}_{t}, P_{t}, e_{t}\right)_{t \in T}$ be an irreducible standard economy. We recall that $E_{+}$denotes the cone $\sum_{i \in I} E_{+}^{i}$ and $E$ denotes the linear space generated by $E_{+}$.

Claim 4.1. The cone $E_{+}$is convex, pointed and closed in $E$.

Proof of Claim 4.1. It is straightforward to check that the set $E_{+}$is convex and pointed. et $\left(a_{n}\right)$ be a sequence in $E_{+}$converging to $a$ in $E$. For each $n \in \mathbb{N}$, there exists $\left(a_{n}^{i}\right)_{i \in I} \in$ $\prod_{i \in I} E_{+}^{i}$ such that $a_{n}=\sum_{i \in I} a_{n}^{i}$. Hence for each $i \in I$, we have $0 \leqslant a_{n}^{i}(\omega, \ell) \leqslant a_{n}(\omega, \ell)$ for every $(\omega, \ell) \in \Omega \times L$. Since the sequence $\left(a_{n}(\omega, \ell)\right)$ converges to $a(\omega, \ell)$ then the sequence $a_{n}^{i}(\omega, \ell)$ is bounded. Passing to a subsequence if necessary, we can assume that there exists $\left(a^{i}\right)_{i \in I} \in \prod_{i \in I} E_{+}^{i}$ such that the sequence $\left(a_{n}^{i}\right)$ converges to $a^{i}$. Therefore $a=\sum_{i \in I} a^{i}$ belongs to $E_{+}$. 
Following the notations in Cornet, Topuzu, and Yildiz (2003), we consider the following coalitional production economy

$$
\mathscr{E}_{C}=\left\{E,(T, \Sigma, \mu),\left(X_{t}, \succ_{t}, e_{t}, Y(t)\right)_{t \in T}\right\}
$$

where $C=E_{+}, \succ_{t}$ is the binary relation defined by $P_{t}$ and $Y(t)=-C$ for every $t \in T$. Applying Corollary 3.1 in Cornet, Topuzu, and Yildiz $(2003)^{16}$ there exists a triple $(x, z, \pi)$ where $x \in \mathscr{S}_{X}$ is a private assignment, $z \in-C$ and $\pi: E \rightarrow \mathbb{R}$ is a non-zero linear functional such that

(a) for $\mu$-a.e. $t \in T, \pi\left(x_{t}\right) \leqslant \pi\left(e_{t}\right)$ and $y \in P_{t}\left(x_{t}\right)$ implies $\pi(y) \geq \pi\left(x_{t}\right)$;

(b) $z \in \operatorname{argmax}\left\{\pi\left(z^{\prime}\right): z^{\prime} \in-C\right\}$; and

(c) $\int_{T} x d \mu=\int_{T} e d \mu+z$.

From (b) it is immediate that $\pi(z)=0$ (since $-C$ is a cone). From this, (a), and (c), $\pi\left(x_{t}\right)=\pi\left(e_{t}\right)$ for almost all $t$ follows directly. Moreover it follows from (b) that $\pi_{\mid E_{+}} \geq 0$ in the sense that for every $y \in E_{+}$, we have $\pi(y) \geq 0$.

We claim that $\pi(e(T))>0$. Indeed, assume by way of contradiction that $\pi(e(T))=0$. From Assumption (S.2), there exists $a^{i} \in E_{++}^{i}$ for each $i$ such that $e(T)=\sum_{i \in I} a^{i}$. Since $\pi_{\mid E_{+}} \geq 0$, it follows that $\pi_{\mid E_{+}^{i}}=0$ for each $i$, implying the contradiction $\pi=0$. Let $A=\{t \in$ $\left.T: \pi\left(e_{t}\right)>0\right\}$, this set belongs to $\Sigma$ and $\mu(A)>0$.

Claim 4.2. For every $t \in A$, $y \in P_{t}\left(x_{t}\right)$ implies $\pi(y)>\pi\left(e_{t}\right)$.

Proof. Let $t \in A$ and $y \in P_{t}\left(x_{t}\right)$. From property (a) we already know that $\pi(y) \geq \pi\left(e_{t}\right)$. Assume by way of contradiction that $\pi(y)=\pi\left(e_{t}\right)$. Since $P_{t}\left(x_{t}\right)$ is open in $X_{t}$ there exists $\alpha \in(0,1)$ such that $\alpha y \in P_{t}\left(x_{t}\right)$. Then applying property (a) we get $\alpha \pi(y) \geq \pi\left(e_{t}\right)$. This yields a contradiction since $\pi\left(e_{t}\right)>0$.

Claim 4.3. The set $A$ is of full measure, i.e., $\mu(A)=1$.

Proof of Claim 4.3. Assume by way of contradiction that $B:=T \backslash A$ is such that $\mu(B)>0$. Let $\tilde{x}: T \rightarrow E_{+}$be defined by

$$
\forall t \in T^{i}, \quad \tilde{x}_{t}=x_{t}-\frac{1}{\mu\left(T^{i}\right)} z^{i} .
$$

The function $\tilde{x}$ is a private assignment, i.e., $\tilde{x} \in \mathscr{S}_{X}$, it satisfies $\pi\left(\tilde{x}_{t}\right)=\pi\left(x_{t}\right)=\pi\left(e_{t}\right)$, $P_{t}\left(\tilde{x}_{t}\right) \subset P_{t}\left(x_{t}\right)$ and it is feasible. Then applying the irreducibility condition to $\tilde{x}$, there exist two private assignments $y, w \in \mathscr{S}_{X}$ such that

$$
\int_{A} \tilde{x} d \mu+\int_{B} e d \mu=\int_{A} y d \mu+\int_{B} w d \mu
$$

and

$$
y_{t} \in P_{t}\left(\tilde{x}_{t}\right) \quad \text { for } \mu \text {-a.e. } t \in A \text {. }
$$

\footnotetext{
${ }^{16}$ In Cornet, Topuzu, and Yildiz (2003) the space $E$ is an Euclidean space $\mathbb{R}^{H}$ for some finite set $H$. But their result can be straightforwardly generalized to any finite dimensional linear space.
} 
Since $\pi\left(e_{t}\right)=0$ for $\mu$-a.e. $t \in B$, we get

$$
\int_{A} \pi\left(\tilde{x}_{t}\right) \mu(d t)=\int_{A} \pi\left(y_{t}\right) \mu(d t)+\int_{B} \pi\left(w_{t}\right) \mu(d t) .
$$

Since $y_{t} \in P_{t}\left(\tilde{x}_{t}\right)$ we get from Claim 4.2 that $\pi\left(y_{t}\right)>\pi\left(e_{t}\right)=\pi\left(\tilde{x}_{t}\right)$ for $\mu$-a.e. $t \in A$, and since $\pi_{\mid E_{+}} \geq 0$ we have $\pi\left(w_{t}\right) \geq 0$. This implies that

$$
\int_{A} \pi\left(\tilde{x}_{t}\right) \mu(d t)=\int_{A} \pi\left(y_{t}\right) \mu(d t)+\int_{B} \pi\left(w_{t}\right) \mu(d t)>\int_{A} \pi\left(\tilde{x}_{t}\right) \mu(d t)
$$

which yields a contradiction.

Since preference relations are strictly monotone, we get from Claims 4.2 and 4.3 that

$$
\forall i \in I, \quad \forall y \in E_{+}^{i}, \quad y \neq 0 \Longrightarrow \pi(y)>0 .
$$

Since $\pi\left(z^{i}\right)=0$ we get that for each $i \in I, z^{i}=0$ and $z=0$. We have thus proved that the feasibility constraints is satisfied with an (exact) equality, i.e., $\int_{T} x d \mu=\int_{T} e d \mu$.

The linear functional $\pi$ can be extended to $\mathbb{R}^{\Omega \times L}$. In particular, there exists a function $p: \Omega \rightarrow \mathbb{R}^{L}$ such that

$$
\forall y \in E, \quad \pi(y)=\mathbb{E}[p \cdot y] .
$$

It is now straightforward to prove that $(x, p)$ is a competitive equilibrium of the economy $\mathscr{E}$.

\section{Core allocations}

The notion below is a variant of the private core introduced in Yannelis (1991).

Definition 5.1. A feasible private assignment $x \in \mathscr{S}_{X}$ is a private weak core allocation for the economy $\mathscr{E}$ if there do not exist a coalition $S \in \Sigma$ with $\mu(S)>0$ and a private assignment $y$ such that $y$ is feasible for the coalition $S$, i.e.,

$$
\int_{S} y_{t} \mu(d t)=\int_{S} e_{t} \mu(d t)
$$

and for $\mu$-a.e. $t \in S$, the plan $y_{t}$ is strictly preferred to $x_{t}$, i.e., $y_{t} \in P_{t}\left(x_{t}\right)$.

The equivalence theorem in Aumann (1964) still prevails in the framework of differential information.

Theorem 5.1. The sets of competitive and private weak core allocations coincide.

Proof of Theorem 5.1. Clearly, any competitive allocation belongs to the private weak core. To verify the converse, suppose $x \in \mathscr{S}_{X}$ is a private weak core allocation. Let $\varphi: T \rightarrow 2^{E}$ be the correspondence given by

$$
\forall t \in T, \quad \varphi(t)=P_{t}\left(x_{t}\right) \cup\left\{e_{t}\right\} .
$$


Then the set $\int_{T} \varphi d \mu$ is non-empty ${ }^{17}$ and convex because the measure space $(T, \Sigma, \mu)$ is atomless (see e.g. (Hildenbrand, 1974, Theorem 2)). Moreover, because $x$ belongs to the private weak core,

$$
\left[\{e(T)\}-E_{++}^{c}\right] \bigcap \int_{T} \varphi d \mu=\emptyset
$$

where we recall that $E_{++}^{c}$ is the space of $\mathscr{F}^{c}$-measurable functions from $\Omega$ to $\mathbb{R}_{++}^{L}$. Indeed, suppose the contrary. Then there are a $v \in E_{++}^{c}$ (in particular $v \neq 0$ ) and an integrable function $g: T \rightarrow E$ such that $g(t) \in \varphi(t)$ for $\mu$-a.e. $t \in T$ and

$$
v+\int_{T} g(t) \mu(d t)=\int_{T} e_{t} \mu(d t) .
$$

Set $S=\left\{t \in T: g(t) \in P_{t}\left(x_{t}\right)\right\}$. By Assumption (S.3), the set $S$ belongs to $\Sigma$. By definition of $\varphi$ we have $g(t)=e_{t}$ for $\mu$-a.e. $t \in T \backslash S$ and hence

$$
v+\int_{S} g(t) \mu(d t)=\int_{S} e_{t} \mu(d t) .
$$

In particular, since $v \neq 0$, we must have $\mu(S)>0$. Let $\widetilde{g}: T \rightarrow E$ be given by

$$
\widetilde{g}(t)=g(t)+\frac{1}{\mu(S)} v .
$$

Observe first that $\widetilde{g}$ is a private assignment since $E_{+}^{c}$ is a subset of $E_{+}^{i}$ for every information type $i$. Then $\int_{S} \widetilde{g} d \mu=\int_{S} e d \mu$, i.e., the assignment $\widetilde{g}$ is feasible for the coalition $S$. Moreover, $\widetilde{g}(t) \in P_{t}(g(t))$ for $\mu$-a.e. $t \in S$ because preferences are strictly monotone, whence $\widetilde{g}(t) \in$ $P_{t}\left(x_{t}\right)$ by transitivity. This contradicts the fact that $x$ is a private weak core allocation.

It follows now from the separation theorem that there is a non-zero linear functional $\pi: E \rightarrow \mathbb{R}$ such that

$$
\forall \xi \in \int_{T} \varphi d \mu, \quad \pi(\xi) \geq \pi(e(T))
$$

and $\pi(y) \geq 0$ for every $y \in E_{+}^{c}$. Following almost verbatim the arguments ${ }^{18}$ in Hildenbrand (1974), we can prove that for $\mu$-a.e. $t \in T$,

$$
y \in P_{t}\left(x_{t}\right) \Longrightarrow \pi(y) \geq \pi\left(e_{t}\right) \text { and } \pi\left(x_{t}\right)=\pi\left(e_{t}\right) .
$$

Since preferences are strictly monotone, we deduce that for each information type $i$, we have $\pi(z) \geq 0$ for each $z \in E_{+}^{i}$. Thus we must have $\pi(e(T))>0$ since $e(T) \in \sum_{i \in I} E_{++}^{i}$ and $\pi$ is not zero. The end of the proof is omitted since it follows almost verbatim the end of the proof of Theorem 4.1.

When preference relations are represented by expected utilities, we may consider the notion of private core allocations.

\footnotetext{
${ }^{17}$ For instance $\int_{T} e_{t} \mu(d t)$ belongs to this set.

${ }^{18}$ In particular applying (Hildenbrand, 1974, Proposition 6).
} 
Definition 5.2. Assume that preference relations are represented by expected utilities. A feasible private assignment $x \in \mathscr{S}_{X}$ is a private core allocation for the economy $\mathscr{E}$ if there do not exist a coalition $S \in \Sigma$ with $\mu(S)>0$ and a private assignment $y$ such that $y$ is feasible for the coalition $S$, i.e.,

$$
\int_{S} y_{t} \mu(d t)=\int_{S} e_{t} \mu(d t)
$$

and

$$
\mu\left\{t \in S: h_{t}\left(y_{t}\right) \geq h_{t}\left(x_{t}\right)\right\}=\mu(S) \text { and } \mu\left\{t \in S: h_{t}\left(y_{t}\right)>h_{t}\left(x_{t}\right)\right\}>0 .
$$

Obviously, a private core allocation is a private weak core allocation. The equivalence theorem is still valid.

Theorem 5.2. Assume that preference relations are represented by expected utilities. The sets of competitive and private core allocations coincide.

Proof. Clearly, a private core allocation is a private weak core allocation. Applying Theorem 5.1, it is also a competitive allocation. To verify the converse, suppose $x \in \mathscr{S}_{X}$ is a competitive allocation and suppose it is not a private core allocation. Then there exist a coalition $S \in \Sigma$ with $\mu(S)>0$ and a private assignment $y$ such that $y$ is feasible for the coalition $S$, i.e.,

$$
\int_{S} y_{t} \mu(d t)=\int_{S} e_{t} \mu(d t)
$$

and

$$
\mu\left\{t \in S: h_{t}\left(y_{t}\right) \geq h_{t}\left(x_{t}\right)\right\}=\mu(S) \text { and } \mu\left\{t \in S: h_{t}\left(y_{t}\right)>h_{t}\left(x_{t}\right)\right\}>0 .
$$

Set $S_{+}=\left\{t \in S: h_{t}\left(y_{t}\right) \geq h_{t}\left(x_{t}\right)\right\}$ and $S_{++}=\left\{t \in S: h_{t}\left(y_{t}\right)>h_{t}\left(x_{t}\right)\right\}$. Let $p: \Omega \rightarrow \mathbb{R}^{L}$ be a non-zero price such that $(x, p)$ is a competitive equilibrium. For $\mu$-a.e. $t \in S_{++}$, the contingent plan $x_{t}$ is optimal in the budget set $B_{t}(p)$, implying that $\mathbb{E}\left[p \cdot y_{t}\right]>\mathbb{E}\left[p \cdot e_{t}\right]$. For every $\varepsilon>0$ and for every $t$ in $S_{+}, y_{t}+\varepsilon \mathbf{1} \in P_{t}\left(x_{t}\right)$ since preference relations are strictly monotone and transitive. ${ }^{19}$ It follows that for $\mu$-a.e. $t \in S_{+}, \mathbb{E}\left[p \cdot y_{t}\right]+\varepsilon \mathbb{E}[p \cdot 1]>\mathbb{E}\left[p \cdot e_{t}\right]$. Letting $\varepsilon$ tend to 0 , we get $\mathbb{E}\left[p \cdot y_{t}\right] \geq \mathbb{E}\left[p \cdot e_{t}\right]$ for $\mu$-a.e. $t \in S_{+}$. Since $\mu\left(S_{++}\right)>0$, we get

$$
\begin{aligned}
\mathbb{E}\left[p \cdot \int_{S} y d \mu\right] & =\int_{S} \mathbb{E}\left[p \cdot y_{t}\right] \mu(d t) \\
& =\int_{S_{++}} \mathbb{E}\left[p \cdot y_{t}\right] \mu(d t)+\int_{S \backslash S_{++}} \mathbb{E}\left[p \cdot y_{t}\right] \mu(d t) \\
& >\int_{S_{++}} \mathbb{E}\left[p \cdot e_{t}\right] \mu(d t)+\int_{S \backslash S_{++}} \mathbb{E}\left[p \cdot e_{t}\right] \mu(d t) \\
& >\int_{S} \mathbb{E}\left[p \cdot e_{t}\right] \mu(d t) \\
& >\mathbb{E}\left[p \cdot \int_{S} e d \mu\right]
\end{aligned}
$$

This contradicts the feasibility of the assignment $y$ for the coalition $S$.

\footnotetext{
${ }^{19}$ The contingent plan $\mathbf{1}$ is the function $\mathbf{1}: \Omega \rightarrow \mathbb{R}^{L}$ defined by $\mathbf{1}(\omega)=\mathbf{1}_{L}$ for every $\omega \in \Omega$.
} 
We now adapt the definition of the weak fine core introduced by Yannelis (1991) and Koutsougeras and Yannelis (1993) to the framework of exact feasibility constraints. We first observe that without any loss of generality, we can assume that the coarsest $\sigma$-algebra containing each $\mathscr{F}^{i}$ coincides with $2^{\Omega}$. Now if $J$ is a subset of $I$, we denote by $\mathscr{F}(J)$ the coarsest $\sigma$-algebra containing each $\mathscr{F}^{j}, j \in J$. In particular we have $\mathscr{F}(\{i\})=\mathscr{F}^{i}$ and $\mathscr{F}(I)=2^{\Omega}$. Similarly, if $S \in \Sigma$ is a coalition then we denote by $\mathscr{F}(S)$ the $\sigma$-algebra $\mathscr{F}(I(S))$ where

$$
I(S):=\left\{i \in I: \mu\left(T^{i} \cap S\right)>0\right\} .
$$

The set $I(S)$ represents the informational types that are present in the coalition $S$ and the $\sigma$-algebra $\mathscr{F}(S)$ represents the information available to each agent of $S$ if they share their information.

Definition 5.3. A feasible assignment $x$ is a weak fine core allocation for the economy $\mathscr{E}$ if there do not exist a coalition $S \in \Sigma$ and an assignment $y$ such that

1. for $\mu$-a.e. $t \in S$, the function $y_{t}$ is $\mathscr{F}(S)$-measurable,

2 . the assignment $y$ is feasible for the coalition $S$, i.e.,

$$
\int_{S} y_{t} \mu(d t)=\int_{S} e_{t} \mu(d t),
$$

3. for $\mu$-a.e. $t \in S$, the plan $y_{t}$ is strictly preferred to $x_{t}$, i.e., $y_{t} \in P_{t}\left(x_{t}\right)$.

Observe that no measurability constraints are imposed on a weak fine core allocation. As in Einy, Moreno, and Shitovitz (2001), if we can extend the preference relations then the weak fine core of an economy $\mathscr{E}$ coincides (and is thus non-empty) with the private core of the symmetrized economy $\mathscr{E}^{*}$.

Definition 5.4. The preference relations of an economy $\mathscr{E}$ are said extendable if for $\mu$-a.e. $t \in T$, there exists a correspondence $P_{t}^{*}$ from $\mathbb{R}_{+}^{\Omega \times L}$ into $\mathbb{R}_{+}^{\Omega \times L}$ such that

1. the preference relations defined by $P^{*}$ are measurable, irreflexive, transitive, continuous and strictly monotone;

2. the correspondence $P_{t}^{*}$ extends $P_{t}$ in the sense that $P_{t}(x) \subset P_{t}^{*}(x)$ for every $x \in X_{t}$.

Remark 5.1. If the preference relations are represented by expected utility functions then they are automatically extendable.

Definition 5.5. If $\mathscr{E}$ is an economy with extendable preference relations, then we let $\mathscr{E}^{*}$ be the economy defined by

$$
\mathscr{E}^{*}=\left(\mathscr{F}_{t}^{*}, P_{t}^{*}, e_{t}\right)_{t \in T}
$$

where $\mathscr{F}_{t}^{*}=2^{\Omega}$ for every $t \in T$. The economy $\mathscr{E}^{*}$ is called the symmetrization of $\mathscr{E}$.

Observe that the economy $\mathscr{E}^{*}$ is symmetric in the sense that every agent has the same information. The proof of the following theorem is based on a result by Vind (1968) and follows almost verbatim the proof of Proposition 5.1 in Einy, Moreno, and Shitovitz (2001).

Theorem 5.3. If $\mathscr{E}$ is an irreducible economy with extendable preferences then the weak fine core of $\mathscr{E}$ coincides with the private core of the symmetrized economy $\mathscr{E}^{*}$.

Remark 5.2. It follows as a corollary of Theorems 4.1, 5.1 and 5.3 that the weak fine core of an irreducible economy with extendable preferences is non-empty. 


\section{Contract enforcement and incentive compatibility}

A competitive allocation as well as a private core allocation are ex-ante solutions corresponding to actions taken at $\tau=0$. In order to address the issue of execution (or enforcement) of contracts at $\tau=1$, we assume that there is an intermediary (a "government institution" or a "market institution") that is responsible for the execution of contracts. In this section we assume that the family $\left(\mathscr{F}^{i}\right)_{i \in I}$ is common knowledge to agents. We also restrict our attention to preference relations represented by expected utility functions.

At $\tau=1$ a state of nature is realized. If the intermediary is able to identify the true state then he can enforce the receipts and deliveries of commodities specified by the contracts made at the previous date, i.e., each agent $t$ receives the net trade $z_{t}(\omega):=x_{t}(\omega)-e_{t}(\omega)$. This is possible since it follows from the (exact) feasibility constraints that

$$
\int_{t \in T} z_{t}(\omega) \mu(d t)=0
$$

This implies that they are no issues concerning execution of contracts.

More interesting is the situation where the intermediary has an incomplete information concerning the true state of nature. In that case, each agent $t$ has to report his information and claims for the corresponding net trade. However, agents may have incentives to misreport their information. If $\omega$ is the realized state of nature, agent $t$ should report his information, i.e., any state in $E_{t}(\omega),{ }^{20}$ but if for some other state $\sigma_{t}$ we have

$$
x_{t}\left(\sigma_{t}\right)-e_{t}\left(\sigma_{t}\right)+e_{t}(\omega) \in \mathbb{R}_{+}^{L}
$$

and

$$
u_{t}\left(\omega, x_{t}\left(\sigma_{t}\right)-e_{t}\left(\sigma_{t}\right)+e_{t}(\omega)\right)>u_{t}\left(\omega, x_{t}(\omega)\right)
$$

then agent $t$ gains by reporting the (uncorrect) state $\sigma_{t}$. If

$$
\int_{t \in T} z_{t}\left(\sigma_{t}\right) \mu(d t) \neq 0
$$

then the intermediary cannot execute contracts. In general it is not possible to avoid such a situation.

Now assume that there is a legal procedure that agents can use to prove that they are not misreporting their information. Assume that this procedure is costly but that the cost of such an action is repaid by agents whose misreporting can be revealed. This has two consequences: first an agent uses this legal procedure only if he is sure that he can detect a misreporting and second an agent decides to misreport only if he is sure that he cannot be detected by other agents. For this argument to be valid, each agent needs to know the information structure of the others.

Recall that every agent in $T^{i}=\left\{t \in T: \mathscr{F}_{t}=\mathscr{F}^{i}\right\}$ has the same information $\mathscr{F}^{i}$, therefore an agent $t \in T^{i}$ alone cannot misreport a state since all the other agents in $T^{i}$ can detect his misreport. This implies that only a whole coalition $T^{i}$ can misreport. More precisely, agents of information type $i$ have an incentive to misreport the realized event $E^{i}(\omega)$ by announcing the state $\sigma$ if

\footnotetext{
${ }^{20}$ Recall that whatever is the state $a$ in $E_{t}(\omega)$ that agent $t$ reports, he gets the same utility.
} 
1. agents not in $T^{i}$ cannot discern $\sigma$ and any possible state in $E^{i}(\omega)$, i.e., for every $j \neq i$, for $\mu$-a.e. $\tau \in T^{j}$, the set $\{\sigma\} \cup E^{i}(\omega)$ is a subset on an atom of $\mathscr{F}^{j}$;

2. almost every agent in $T^{i}$ has an incentive to announce $\sigma$, i.e., for $\mu$-a.e. $t \in T^{i}$, we have

$$
e_{t}(\omega)+x_{t}(\sigma)-e_{t}(\sigma) \in \mathbb{R}_{+}^{L}
$$

and

$$
u_{t}\left(a, e_{t}(\omega)+x_{t}(\sigma)-e_{t}(\sigma)\right)>u_{t}\left(a, x_{t}(\omega)\right) .
$$

Naturally, there is no reason to restrict coalitions to be composed of agents of the same information type. It may the case that all agents of information type $i_{1}$ and $i_{2}$ have an incentive to commonly misreport the same state $\sigma$ when the realized state is $\omega$. In order to agree to misreport, agents in $T^{i_{1}} \cup T^{i_{2}}$ have first to agree on the set of possible realized states of nature. We assume that agents of type $i_{1}$ don't want to share or reveal information with agents of type $i_{2}$ and vice versa. Therefore they have to agree on common knowledge events. This leads us to the following definition.

Definition 6.1. Let $\omega \in \Omega$, if $i \in I$ is an information type, then we let $E^{i}(\omega)$ be the atomic event in $\mathscr{F}^{i}$ which contains $\omega$. If $J \subset I$ then we denote by $\mathscr{F}^{J}$ the meet $\sigma$-algebra $\wedge_{j \in J} \mathscr{F}^{j}$ which is interpreted as the common knowledge information of the coalition $J$ of types. If $\omega \in \Omega$ we let $E^{J}(\omega)$ be the atomic event in $\mathscr{F}^{J}$ which contains $\omega$.

According to this definition, the common knowledge information of the coalition $S=$ $T^{i_{1}} \cup T^{i_{2}}$, is that the realized state of nature belongs to $E^{\left\{i_{1}, i_{2}\right\}}(\omega)$. Since any agent wants to reveal information to other agents, it must be the case that every agent of the coalition $S$ gains by announcing the state $\sigma$ instead of any possible realized state of nature in the common knowledge event $E^{\left\{i_{1}, i_{2}\right\}}(\omega)$, i.e.,

$$
\forall a \in E^{\left\{i_{1}, i_{2}\right\}}(\omega), \quad\left\{\begin{array}{l}
e_{t}(a)+x_{t}(\sigma)-e_{t}(\sigma) \in \mathbb{R}_{+}^{L} \\
\text { and } \\
u_{t}\left(a, e_{t}(a)+x_{t}(\sigma)-e_{t}(\sigma)\right)>u_{t}\left(a, x_{t}(a)\right) .
\end{array}\right.
$$

If agents in $S=T^{i_{1}} \cup T^{i_{2}}$ decide to misreport by announcing $\sigma$ instead of any state in $E^{\left\{i_{1}, i_{2}\right\}}(\omega)$, they must check if any agent $\tau \notin S$ can detect that $\sigma$ is not the realized state of nature. ${ }^{21}$ But to do so, agents in the coalition $S$ need to agree on what is agent $\tau$ 's information. The common knowledge of the coalition $S$ is that any state $a \in E^{\left\{i_{1}, i_{2}\right\}}(\omega)$ is a possible candidate for the realized state of nature. Therefore, they must check that for every $a \in E^{\left\{i_{1}, i_{2}\right\}}(\omega)$, every agent $\tau \notin S$ cannot discern states $a$ and $\sigma$, i.e.,

$$
E^{\left\{i_{1}, i_{2}\right\}}(\omega) \subset E_{\tau}(\sigma), \quad \text { for } \mu \text {-a.e. } \tau \notin S .
$$

This leads us to the following concept of coalitional incentive compatibility. Actually, this is not a new concept since it is the straightforward adaptation to economies with a continuum of agents of the coalitional incentive compatibility condition introduced by Krasa and Yannelis (1994).

\footnotetext{
${ }^{21}$ In particular, the information structure $\left(\mathscr{F}^{i}\right)_{i \in I}$ must be common knowledge to all agents.
} 
Definition 6.2. A private assignment $x \in \mathscr{S}_{X}$ is said coalitional incentive compatible if there is no coalition $S \in \Sigma$ with $\mu(S) \in(0,1)$ that has an incentive to misreport a state of nature. A coalition $S \in \Sigma$ has an incentive to misreport a state of nature if there exist states $\omega \neq \sigma$ such that

1. for $\mu$-a.e. $\tau \notin S$, agent $\tau$ cannot discern state $\sigma$ and any state $\operatorname{in}^{22} E^{I(S)}(\omega)$, i.e., $\{\sigma\} \cup E^{I(S)}(\omega)$ is a subset of an atom of the information algebra $\mathscr{F}_{\tau}$; in other words

$$
E^{I(S)}(\omega) \subset E_{\tau}(\sigma), \quad \text { for } \mu \text {-a.e. } \tau \notin S \text {; }
$$

2. for $\mu$-a.e. $t \in S$, we have

$$
\forall a \in E^{I(S)}(\omega), \quad\left\{\begin{array}{l}
e_{t}(a)+x_{t}(\sigma)-e_{t}(\sigma) \in \mathbb{R}_{+}^{L} \\
\text { and } \\
u_{t}\left(a, e_{t}(a)+x_{t}(\sigma)-e_{t}(\sigma)\right)>u_{t}\left(a, x_{t}(a)\right) .
\end{array}\right.
$$

Remark 6.1. A necessary condition for a coalition $S \in \Sigma$ to have an incentive to misreport a state is that

$$
I(S) \cap I(T \backslash S)=\emptyset .
$$

Condition (4) comes from the fact that an agent $t$ with information type $i$ cannot misreport a state to another agent $t^{\prime}$ whose information type is the same.

Remark 6.2. Definitions 1 and 2 in Krasa and Yannelis (1994) are specified for the case of one commodity. In Section 3.4, they extend the definition to the case of more than one commodity by requiring that a deviation should improve the deviating coalition in a set of states that is common knowledge information to the deviating coalition. This is exactly our condition (3) in Definition 6.2. They don't state explicitly that members of the complementary coalition cannot detect the misreport according to any state in the event that is common knowledge to the deviating coalition (i.e., our condition (2) in Definition 6.2). However, this is the only interpretation that is consistent with the proof and the objective of their definition.

Remark 6.3. A cautious reader will notice that our concept of coalitional incentive compatibility is slightly different than the weak coalitional Bayesian incentive compatibility introduced in Koutsougeras and Yannelis (1993) (see also Hervès-Beloso, Moreno-García, and Yannelis (2005) and Podczeck and Yannelis (2007)) where it is imposed that in order to misreport state $\omega$ by announcing $\sigma$, agents in a coalition $S$ should have the same information about the realized state of nature, i.e., for a.e. $t \in S$

$$
E_{t}(\omega)=E^{I(S)}(\omega), \quad \text { for } \mu \text {-a.e. } t \in S \text {. }
$$

We consider that a coalition could misreport even if they do not have the same information. What matters is that they agree to misreport on the common knowledge information.

\footnotetext{
${ }^{22}$ We recall that for each coalition $E \in \Sigma$, the set $I(E)$ is the set of information types present in the coalition, i.e., $I(E)=\left\{i \in I: \mu\left(E \cap T^{i}\right)>0\right\}$.
} 
Remark 6.4. The coalitional incentive compatibility condition can be stated in terms of interim expected utility instead of ex-post utility. This leads to the concept of coaltional Bayesian incentive compatibility. We refer to Hahn and Yannelis (1997), Hahn and Yannelis (2001) and Glycopantis and Yannelis (2005) for details.

We next show that competitive or core allocation fulfills the coalitional incentive compatibility. Actually, this is result is true for Pareto optimal allocations.

Definition 6.3. A feasible assignment $x \in \mathscr{S}_{X}$ is said Pareto optimal if there does not exist a feasible assignment $y \in \mathscr{S}_{X}$ such that

$$
\mu\left\{t \in T: h_{t}\left(y_{t}\right) \geq h_{t}\left(x_{t}\right)\right\}=1 \quad \text { and } \quad \mu\left\{t \in T: h_{t}\left(y_{t}\right)>h_{t}\left(x_{t}\right)\right\}>0 .
$$

Remark 6.5. It is straightforward to check that competitive and private core allocations are Pareto optimal.

The main motivation in considering (exact) equality in the feasibility constraints is the theorem below where we show that if free disposal is not allowed in the feasibility constraints then every Pareto optimal assignment is coalitional incentive compatibility. A straightforward consequence is that contracts of every competitive or private core allocations are enforceable.

Theorem 6.1. Every (exact) feasible Pareto optimal assignment (and thus every competitive or private core allocation) is coalitional incentive compatible.

Versions of Theorem 6.1 have been proved in Koutsougeras and Yannelis (1993), Krasa and Yannelis (1994) and Hahn and Yannelis (2001). For the sake of completeness, we propose a detailed proof.

Proof of Theorem 6.1. Let $x \in \mathscr{S}_{X}$ be a feasible assignment that is Pareto optimal and assume by way of contradiction that there exist a coalition $S \in \Sigma$ with $\mu(S) \in(0,1)$ and two states $\xi \neq \sigma$ such that

1. for $\mu$-a.e. $t \notin S$, for every $a \in E^{I(S)}(\xi)$, the states $a$ and $\sigma$ are not distinguishable, i.e., $\sigma \in E_{t}(a)$

2. for $\mu$-a.e. $t \in S$, for every $a \in E^{I(S)}(\xi)$, we have

$$
e_{t}(a)+z_{t}(\sigma) \in \mathbb{R}_{+}^{L} \quad \text { and } \quad u_{t}\left(a, e_{t}(a)+z_{t}(\sigma)\right)>u_{t}\left(a, x_{t}(a)\right),
$$

where $z_{t}(\omega):=x_{t}(\omega)-e_{t}(\omega)$ for every $\omega \in \Omega$.

We consider now the function $y: T \rightarrow \mathbb{R}^{\Omega \times L}$ defined by

$$
\forall t \in S, \quad \forall \omega \in \Omega, \quad y_{t}(\omega)=\left\{\begin{array}{lll}
e_{t}(\omega)+z_{t}(\sigma) & \text { if } & \omega \in E^{I(S)}(\xi) \\
x_{t}(\omega) & \text { if } & \omega \notin E^{I(S)}(\xi)
\end{array}\right.
$$

and

$$
\forall t \notin S, \quad \forall \omega \in \Omega, \quad y_{t}(\omega)=x_{t}(\omega) .
$$

The function $y_{t}$ is $\mathscr{F}_{t}$-measurable for $\mu$-a.e. $t \in T$. Moreover for each $\omega \in \Omega$, the vector $y_{t}(\omega)$ belongs to $\mathbb{R}_{+}^{L}$. Therefore $y$ is a private assignment, i.e., $y \in \mathscr{S}_{X}$. We have 
$u_{t}\left(\omega, e_{t}(\omega)+z(\sigma)\right)>u_{t}\left(\omega, x_{t}(\xi)\right)$ for $\mu$-a.e. $t \in S$ and every $\omega \in E^{I(S)}(\xi)$, which implies that $h_{t}\left(y_{t}\right)>h_{t}\left(x_{t}\right)$. We have thus proved that

$$
\mu\left\{t \in T: h_{t}\left(y_{t}\right) \geq h_{t}\left(x_{t}\right)\right\}=1
$$

and

$$
\mu\left\{t \in T: h_{t}\left(y_{t}\right)>h_{t}\left(x_{t}\right)\right\}=\mu(S)>0 .
$$

In order to get a contradiction, it is now sufficient to prove that $y$ is a feasible assignment. We first have that

$$
\forall \omega \in \Omega, \quad \int_{T \backslash S} y(\omega) d \mu=\int_{T \backslash S} x(\omega) d \mu,
$$

and

$$
\forall \omega \notin E^{I(S)}(\xi), \quad \int_{S} y(\omega) d \mu=\int_{S} x(\omega) d \mu
$$

implying that for every $\omega \notin E^{I(S)}(\xi)$ we have

$$
\int_{T} y(\omega) d \mu=\int_{S} x(\omega) d \mu=\int_{T} e(\omega) d \mu
$$

Now fix $a \in E^{I(S)}(\xi)$ then

$$
\int_{S} y(a) d \mu=\int_{S} e(a) d \mu+\int_{S} z(\sigma) d \mu .
$$

Recall that $x$ is a feasible allocation, in particular

$$
\int_{S} z(a) d \mu+\int_{T \backslash S} z(a) d \mu=0=\int_{S} z(\sigma) d \mu+\int_{T \backslash S} z(\sigma) d \mu .
$$

But for $\mu$-a.e. $t \notin S$ we have that $\sigma \in E_{t}(a)$ and then $z_{t}(a)=z_{t}(\sigma)$. As a consequence we get that

$$
\int_{S} z(a) d \mu=\int_{S} z(\sigma) d \mu
$$

which implies that for each $a \in E^{I(S)}(\xi)$,

$$
\int_{S} y(a) d \mu=\int_{S}\{e(a)+z(\sigma)\} d \mu=\int_{S}\{e(a)+z(a)\} d \mu=\int_{S} x(a) d \mu .^{23}
$$

Remark 6.6. Observe that the exact feasibility constraints plays a crucial role in the proof of Theorem 6.1.

\footnotetext{
${ }^{23}$ In this argument it is crucial that for every information type $j \in I(T \backslash S)$ and for every possible state of nature $a \in E^{I(S)}(\xi)$, agents with information type $j$ cannot discern $\sigma$ and $a$.
} 


\section{References}

Arrow, K., AND G. Debreu (1954): "Existence of an equilibrium for a competitive economy," Econometrica, 22, 265-290.

Aumann, R. J. (1964): "Markets with a continuum of traders," Econometrica, 32, 39-50.

(1966): "Existence of a competitive equilibrium in markets with a continuum of traders," Econometrica, 34, $1-17$.

BAlder, E. J., AND C. Hess (1995): “Fatou's lemma for multifunctions with unbounded values," Mathematics of Operations Research, 20, 175-188.

Cornet, B., M. Topuzu, AND A. Yildiz (2003): "Equilibrium theory with a measure space of possibly satiated consumers," J. Math. Econom., 39, 175-196.

Daher, W., V. F. MARTINS-DA-Rocha, AND Y. VAilakis (2007): "Asset market equilibrium with short-selling and differential information," Economic Theory, 32, 425-446.

Einy, E., D. Moreno, AND B. Shitovitz (2001): "Competitive and core allocations in large economies with differential information," Economic Theory, 18, 321-332.

Einy, E., AND B. Shitovitz (2001): "Private value allocations in large economies with differential information," Games and Economic Behaviour, 34, 287-311.

Glycopantis, D., A. Muir, AND N. C. YAnNElis (2002): “On extensive form implementation of contracts in differential information economies," Economic Theory, 21, 495-526.

Glycopantis, D., AND N. C. Yannelis (2005): Differential information economies, Studies in Economic Theory, 19. Berlin Heidelberg New York: Springer.

Hahn, G., AND N. C. YANnelis (1997): "Efficiency and incentive compatibility in differential information economies," Economic Theory, 10, 383-411.

- (2001): "Coalitional Bayesian Nash implementation in differential information economies," Economic Theory, $18,485-509$.

Hervès-Beloso, C., V. F. Martins-DA-Rocha, AND P. K. Monteiro (2007): "Equilibrium theory with asymmetric information and infinitely many states," Ensaios Ecônomicos EPGE, Getulio Vargas Foundation.

Hervès-Beloso, C., E. Moreno-García, And N. C. Yannelis (2005): "Characterization and incentive compatibility of Walrasian expectations equilibrium in infinite dimensional commodity spaces," Economic Theory, 26, 361-381.

Hildenbrand, W. (1970): "Existence of equilibria for economies with production and a measure space of consumers," Econometrica, 38, 608-623.

(1974): Core and equilibrium of a large economy. Princeton Univ. Press.

Koutsougeras, L. C., AND N. C. YAnnelis (1993): "Incentive compatibility and information superiority of the core on an economy with differential information," Economic Theory, 3, 195-216.

KRASA, S., AND N. C. YANNELIS (1994): "The value allocation of an economy with differential information," Econometrica, 62, 881-900.

McKenzie, L. W. (1959): "On the existence of general equilibrium for a competitive market," Econometrica, 27, 54-71.

PodczecK, K., AND N. C. YANNELIS (2007): "Equilibrium theory with asymmetric information and with infinitely many commodities," Journal of Economic Theory, doi: 10.1016/j.jet.2007.09.011.

RADNER, R. (1968): "Competitive equilibria under uncertainty," Econometrica, 36, 31-58.

- (1982): "Equilibrium under uncertainty," in Handbook of mathematical economics, vol. II, pp. 923-1006. Amsterdam: North-Holland.

Schmeidler, D. (1970): "Fatou's Lemma in several dimensions," Proceedings of the American Mathematical Society, 24, 300-306.

VIND, K. (1968): "A third remark on the core of an atomless economy," Econometrica, 36, 31-58.

YANnELIS, N. C. (1991): "The core of an economy with differential information," Economic Theory, 1, $183-198$. 